\title{
Die Beschäftigungsgarantie der Unternehmen
}

\author{
Brigitte Tschudi
}

\section{Relevanz}

Arbeitslosenversicherung und Mindestsicherung mindern die Einkommensrisiken, damit die Menschen in allen Lebenslagen einen gleichmäßigen Wohlstand erhalten können. Noch besser ist es, wirtschaftliche Risiken erst gar nicht entstehen zu lassen, indem die Unternehmen das Risiko übernehmen und im Auf und Ab der Konjunktur Beschäftigungs- und Lohnsicherheit gewähren. Während die Gewinne stark schwanken, bleiben Löhne und Beschäftigung weitgehend stabil. Bei Familienunternehmen liegen Eigentum und Management in einer Hand. Sie pflegen ein engeres Vertrauensverhältnis zu ihren Angestellten und bieten daher eine bessere Beschäftigungsgarantie als Firmen im Streubesitz und mit unabhängigen Managern. Sie sind eher in der Lage, ihre Beschäftigten davon zu überzeugen, mit vorübergehenden Lohnzugeständnissen einen Teil des Risikos mitzutragen, damit die Beschäftigungsgarantie auch in schwieriger Lage möglich bleibt. Ein glaubwürdiges Versprechen von Beschäftigungssicherheit hängt zudem von der Finanzierungskraft und dem Zugang zu Krediten ab, um Engpässe besser überbrücken zu können. Diese Beschäftigungsgarantie schützt den Sozialstaat vor Überbeanspruchung und macht krisenrobuste Unternehmen zur wichtigsten Sozialversicherung überhaupt.

Christian Keuschnigg

B. Tschudi $(\bowtie)$

Universität St. Gallen, St. Gallen, Schweiz

E-Mail: brigitte.tschudi@student.unisg.ch

(C) Der/die Autor(en) 2018

C. Keuschnigg (Hrsg.), Inklusives Wachstum und wirtschaftliche Sicherheit, https://doi.org/10.1007/978-3-658-21344-2_18 


\title{
Quelle
}

Der nachfolgende Text ist eine Zusammenfassung von: Ellul, Andrew, Marco Pagano und Fabiano Schivardi (2015), Employment and Wage Insurance Within Firms: Worldwide Evidence, Review of Financial Studies, erscheint demnächst.

Wer eine unselbständige Beschäftigung wählt, möchte einen gleichmäßigen Lohn und sichere Beschäftigung möglichst unabhängig vom Auf und Ab der Konjunktur. Wer Unternehmer ist, braucht dagegen Mut zum Risiko. Wenn in einer Rezession die Umsätze einbrechen und Löhne und andere Kosten weitgehend starr sind, drohen Verluste. Umso höher müssen ihre Gewinne sein, wenn der Absatz boomt. Die hohe Risikobereitschaft der Eigentümer macht es möglich, dass Unternehmen ihren eher risikoscheuen Mitarbeitern das Risiko abnehmen, indem sie ihnen einen von der Wirtschaftslage weitgehend unabhängigen Lohn zahlen und Beschäftigung garantieren. Die Anstellung ist also wie eine stillschweigende Vereinbarung zwischen Unternehmern und Beschäftigten, ohne dass es dazu einen schriftlichen „Versicherungsvertrag“ gäbe. Wie bei jedem Versicherungsgeschäft hat die Versicherung einen Preis und erfordert ein Lohnzugeständnis im Austausch gegen Beschäftigungs- und Lohnsicherheit, genauso wie die Gewinne der Unternehmer und Eigentümer eine Risikoprämie enthalten müssen, um die Übernahme des Risikos zu entschädigen.

\begin{abstract}
„The family business in Warroad, Minnesota, that didn't lay off a single one of their four thousand employees during this recession, even when their competitors shut down dozens of plants, even when it meant the owners gave up some perks and pay ... understood their biggest asset was the community and the workers who helped build that business ..." (Präsident Obama, nach Baltimore Sun, 06.09.2012).
\end{abstract}

Die stillschweigende Vereinbarung der Unternehmen mit ihren Mitarbeitern fußt auf zwei Pfeilern. Erstens muss das Versprechen von Lohn- und Beschäftigungssicherheit glaubwürdig sein. Die Unternehmen müssen in der Lage sein und es muss sich für sie auszahlen, es nicht bei der ersten auftretenden Schwierigkeit $\mathrm{zu}$ brechen, sondern dieses Versprechen auch unter widrigen Umständen einzuhalten. Zweitens, müssen die Arbeitnehmer eine Wertschätzung für Lohn- und Beschäftigungssicherheit haben, was vom Grad ihrer Risikoscheu abhängt. Und es muss ein hoher Bedarf nach dieser dezentralen Versicherungslösung bestehen, weil z. B. die staatliche Arbeitslosenversicherung und andere Sozialleistungen in Notlagen wenig großzügig sind.

Familienunternehmen, bei denen Management und Eigentum in einer Hand liegen, haben meist ein engeres Verhältnis zu ihren Angestellten als große Unternehmen mit unabhängigen Managern und im Streubesitz. Das führt zu zwei empirisch 
überprüfbaren Hypothesen darüber, in welchem Ausmaß Unternehmen tatsächlich Lohn- und Beschäftigungssicherheit bieten. Erstens, Familienunternehmen garantieren sowohl mehr Beschäftigungs- als auch mehr Lohnsicherheit als andere Firmen. Die Besitzerfamilien haben meist über viele Jahre Zeit und Ressourcen investiert, um eine glaubwürdige Reputation aufzubauen und das Vertrauen der Angestellten zu gewinnen. Daher sind sie besser in der Lage, sich selbst glaubwürdig zu verpflichten, den stillschweigenden Versicherungsvertrag einzuhalten, und scheuen auch in schwierigen Situationen davor zurück, dieses Vertrauen aufs Spiel zu setzen. Zweitens können Familienunternehmen beim Auftreten großer negativer Schocks ihre Beschäftigten besser davon überzeugen, sich am Risiko zu beteiligen, indem sie vorübergehende Lohnzugeständnisse machen. Damit bringen sie die Unternehmen oft erst in die Lage, ihre Mitarbeiter trotz verschlechterter Wirtschaftslage weiter zu beschäftigen und können betriebsnotwendige Kündigungen vermeiden. Nach dieser Hypothese garantieren Familienunternehmen mehr Beschäftigungs- und weniger Lohnsicherheit, und nicht beides gleichzeitig, wie die erste Hypothese behauptet.

Familienunternehmen bieten mehr Beschäftigungssicherheit und können ihre Angestellten in schwierigen Situationen eher überzeugen, mit vorübergehenden Lohnzugeständnissen dazu beizutragen.

Neben diesen beiden Hypothesen sind auch andere Faktoren für die Versicherungsleistung der Unternehmen wichtig. Der Bedarf nach einer Beschäftigungs- und Lohngarantie der Unternehmen hängt auch davon ab, wie dicht das staatliche Versicherungsnetz gespannt ist. In Ländern mit einer großzügig ausgebauten Arbeitslosenversicherung und Sozialhilfe für Langzeitarbeitslose wird eine dezentrale Versicherungslösung weniger gebraucht. Außerdem hängt die Fähigkeit der Unternehmen zu dieser Versicherungsleistung von ihrer Eigenkapitalausstattung und ihren Reserven und vom Zugang zu Krediten ab, um Finanzierungsengpässe überbrücken zu können.

Die Forschungsarbeit beruht auf Daten von 7710 Firmen - davon sind ca. $30 \%$ Familienunternehmen - aus 41 Staaten über einen Zeitraum von 25 Jahren. Sie untersucht, wie Umsatzrückgänge in einer Branche auf Beschäftigung und Löhne in den Unternehmen durchschlagen. Es zeigt sich, dass ein Umsatzrückgang um $10 \%$ die Beschäftigung in Nicht-Familienunternehmen je nach Szenario und Spezifikation um 1,2 bis 1,9\% senkt. Dagegen erweist sich die Beschäftigung in Familienunternehmen weitestgehend unabhängig von den Umsatzschwankungen der Branche. Vor allem die Familienunternehmen sind daher in der Lage, ihren Angestellten weitgehende Beschäftigungssicherheit zu garantieren.

Dies ändert sich allerdings, wenn das Ausmaß an staatlicher Versicherung gemessen an der Ersatzquote der Arbeitslosenversicherung in den ersten beiden 
Jahren der Erwerbslosigkeit miteinbezogen wird. Da Nicht-Familienunternehmen deutlich weniger Beschäftigungssicherheit anbieten, haben auch Unterschiede in der staatlichen Arbeitslosenversicherung keinen statistisch signifikanten Einfluss darauf. Die Beschäftigungsgarantie in Familienunternehmen wird hingegen erkennbar beeinflusst. Aus den Schätzergebnissen lässt sich ableiten, dass Familienunternehmen ihren Angestellten eine fast vollständige Beschäftigungsgarantie anbieten würden, wenn die Ersatzquote der Arbeitslosenversicherung Null wäre und damit die staatliche Versicherung vollständig fehlen würde. Wenn jedoch die staatliche Versicherung mit einer Ersatzquote von Eins das Risiko vollständig absichern würde, dann würden die Familienunternehmen keine Beschäftigungsgarantie mehr anbieten und sich nur mehr unwesentlich von den anderen Firmen unterscheiden. Umsatzschwankungen der Branche würden auch bei Familienunternehmen auf das Beschäftigungsniveau durchschlagen.

Wenn der Branchenumsatz um $10 \%$ einbricht, sinkt die Beschäftigung in Nicht-Familienunternehmen zwischen 1,2 bis 1,9\%, während sie in Familienunternehmen im Wesentlichen konstant bleibt.

Beschäftigungssicherheit ist vor allem in der Krise gefragt. Die Studie macht deutlich, dass Familienunternehmen bei negativen Entwicklungen die Beschäftigungsgarantie aufrechterhalten, aber bei Umsatzzuwächsen die Beschäftigung ausweiten. Außerdem zeigt sich, dass eine Beschäftigungsgarantie nur bei vorübergehenden Schocks möglich ist, während ein dauerhafter Umsatzrückgang weder durch Familien- noch durch Nicht-Familienunternehmen versicherbar ist. Dementsprechend kann der Ausbau der staatlichen Sozialversicherung eine dezentrale Versicherungsleistung der Familienunternehmen nur bei vorübergehenden Schocks verdrängen. Schließlich zeigt die Untersuchung, dass Familienunternehmen eine Beschäftigungsgarantie nur aufrechterhalten können, wenn sie über genügend Selbstfinanzierungskraft verfügen und ungehinderten Zugang zu externer Finanzierung haben. Bei einer Kreditverknappung oder einer angespannten Finanzierungslage mit firmenspezifischen Ursachen können Familienunternehmen die Beschäftigungsgarantie nicht mehr leisten und verhalten sich ähnlich wie die Nicht-Familienunternehmen.

Risikoscheue Arbeitnehmer wollen neben Beschäftigungssicherheit auch Lohnsicherheit. Im Allgemeinen herrscht ein hohes Maß an Lohnrigidität. Wenn die Umsätze einbrechen, passen die Unternehmen eher die Zahl ihrer Angestellten als den durchschnittlichen Reallohn an. Betrachtet man alle Unternehmen, dann fällt der durchschnittliche Reallohn um ca. 0,5 bis 0,6\%, wenn der Branchenumsatz um $10 \%$ zurückgeht. Die Reallöhne sind also deutlich stabiler als die Beschäftigung. In den Familienunternehmen werden die Reallöhne jedoch stärker 
angepasst und fallen um 0,7 bis $0,9 \%$. Familienunternehmen bieten also eine überdurchschnittliche Beschäftigungssicherheit, aber dafür geringere Lohnsicherheit an. Offensichtlich können aufgrund des engeren Vertrauensverhältnisses die Unternehmensleitungen ihre Angestellten besser davon überzeugen, über Lohnzugeständnisse einen Teil des Risikos mitzutragen, um damit das Risiko eines Arbeitsplatzverlustes abzubauen.

Wenn der Branchenumsatz um $10 \%$ einbricht, sinkt der Reallohn im Durchschnitt der Unternehmen um 0,5 bis 0,6\%, jedoch um 0,7 bis $0,9 \%$ in Familienunternehmen.

Jede Versicherung hat einen Preis. Auch die Übernahme des Einkommensrisikos durch die Unternehmen ist nicht gratis. Wie hoch fällt nun dieser Preis für die von den Familienunternehmen versprochene Beschäftigungssicherheit aus? Die Untersuchung zeigt, dass Familienunternehmen um 6 bis $9 \%$ tiefere Löhne zahlen als andere Firmen. Ob dieser Lohnunterschied ausschließlich als Preis für die gebotene Beschäftigungssicherheit gedeutet werden kann oder auf andere, unbeobachtete Unterschiede in den persönlichen Fähigkeiten zwischen Angestellten von Familienunternehmen und anderen Firmen zurückzuführen ist, ist nicht mit absoluter Sicherheit geklärt. Für die Annahme, dass es sich beim Lohnabschlag um eine Art Versicherungsprämie handelt, spricht jedoch folgendes Ergebnis. Wenn die Ersatzquote der öffentlichen Arbeitslosenversicherung höher ist, fällt der Lohnabschlag in den Familienunternehmen statistisch signifikant geringer aus. Eine dichter gestrickte staatliche Versicherung reduziert den Wert und den Bedarf nach einer dezentralen Versicherung durch die Unternehmen.

Open Access Dieses Kapitel wird unter der Creative Commons Namensnennung 4.0 International Lizenz (http://creativecommons.org/licenses/by/4.0/deed.de) veröffentlicht, welche die Nutzung, Vervielfältigung, Bearbeitung, Verbreitung und Wiedergabe in jeglichem Medium und Format erlaubt, sofern Sie den/die ursprünglichen Autor(en) und die Quelle ordnungsgemäß nennen, einen Link zur Creative Commons Lizenz beifügen und angeben, ob Änderungen vorgenommen wurden.

Die in diesem Kapitel enthaltenen Bilder und sonstiges Drittmaterial unterliegen ebenfalls der genannten Creative Commons Lizenz, sofern sich aus der Abbildungslegende nichts anderes ergibt. Sofern das betreffende Material nicht unter der genannten Creative Commons Lizenz steht und die betreffende Handlung nicht nach gesetzlichen Vorschriften erlaubt ist, ist für die oben aufgeführten Weiterverwendungen des Materials die Einwilligung des jeweiligen Rechteinhabers einzuholen.

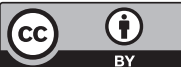

